\title{
Conservative Management of Pharyngo-Cutaneous Fistula Following Traditional Tonsillotomy: A Case Report
}

\author{
Abdullahi Musa Kirfi ${ }^{1 *}$, Shirama Bababa Yakubu ${ }^{2}$ and Auwal Babayo Kwankiyel ${ }^{1}$ \\ ${ }^{1}$ Department of Otorhinolaryngology, Head and Neck Surgery, Abubakar Tafawa Balewa University Teaching Hospital, Bauchi \\ ${ }^{2}$ Department of Radiology, Abubakar Tafawa Balewa University Teaching Hospital, Bauchi
}

*Corresponding author: Abdullahi Musa Kirfi, Department of Otorhinolaryngology, Abubakar Tafawa Balewa University Teaching Hospital Bauchi, Bauchi State, Nigeria.

Received Date: August 14, 2021

Published Date: August 20, 2021

\begin{abstract}
Pharyngo-cutaneous fistula is a communication between the pharynx and the skin. It may occur as a result of surgical procedure, deep neck space infections and malignancies of the upper aerodigestive tract. It may also result from perforation by an upper aerodigestive foreign body. We present a 26 year old housewife and mother of 2, presented to our facility with complaints of anterior neck opening discharging pus and food particles following traditional tonsillotomy. Examination revealed chronically ill-looking woman, weak, lying on couch, wasted, dehydrated. Poor oro-dental hygiene, enlarged and inflamed left tonsil, shattered right tonsil with a cavity at the inferior pole exuding pus. Neck examination revealed dyschromic anterior neck skin, features of dermatitis, a supra-sternal wound measuring $2 \times 2 \mathrm{~cm}$ exuding pus. An assessment of Pharyngo-cutaneous fistula from traditional tonsillotomy was made. Patient was resuscitated, placed on intravenous antibiotics, nasogastric intubation for feeding and daily wound dressing. Full blood count, electrolytes and urea as well as barium fistulography was done. Packed cell volume of $32.1 \%$, White blood cell count of 11.9x109/L, Neutrophilia of 81.6\%, platelets count of 141x109/L. Electrolytes, Urea and Creatinine revealed hyponatremia of $133 \mathrm{mMol} / \mathrm{L}$, hypokalaemia of $2.8 \mathrm{mMol} / \mathrm{L}$, other parameters were normal. Retroviral screening was non-reactive. Barium fistulography revealed numerous fistulous tracts from the oropharynx, the largest being $13 \times 1.5 \mathrm{~cm}$. The airways appear intact. Fistula became closed three weeks after commencement of conservative management. We conclude that pharyngocutaneous fistula from traditional tonsillotomy can be managed conservatively with nasogastric intubation, intravenous antibiotics, wound care, fluid and electrolyte replacement as well as nutritional rehabilitation.
\end{abstract}

Keywords: Pharyngo-cutaneous; Fistula; Traditional; Tonsillotomy

\section{Introduction}

Pharyngo-cutaneous fistula is a communication between the pharynx and the skin. It may occur as a result of surgical procedure, deep neck space infections and malignancies of the upper aerodigestive tract [1-3]. It may also result from perforation by an upper aerodigestive foreign body [4]. Our patient is a 26-yearold housewife and a mother of two. She presented to our facility with complaints of anterior neck opening discharging pus and food particles 8 days after an intervention by a traditional barber who burst her tonsils when she had sore throat characterized by odynophagia, fever and neck swellings. She was said to have bled from the oropharynx, which stopped later on. She developed progressive neck swelling which later on involved the anterior chest wall, for which she was seen at a peripheral hospital, admitted and discharged after seven days with no improvement in her condition. A day after being discharged from the hospital, the neck swelling bursted, draining pus and whatever the patient swallows, associated with fever and generalized body weakness. On account of the above, the patient presented to our facility. 
On examination, she was found to be chronically ill-looking, weak, lying on couch (could not sit), wasted, dehydrated and drooling saliva. Oral cavity and oropharyngeal examination revealed poor oro-dental hygiene, enlarged and inflamed left tonsil, shattered right tonsil with a cavity at the inferior pole exuding purulent material. Neck examination revealed dyschromic anterior neck skin, features of dermatitis, a supra-sternal wound measuring $2 \times 2 \mathrm{~cm}$ exuding pus. Respiratory rate was 24 cycles per minute (cpm), had some transmitted sounds on both lung fields, pulse rate was 64 beats per minute (bpm) with a blood pressure (BP) of 60/40 mmHg. An assessment of pharyngo-cutaneous fistula secondary to traditional tonsillotomy was made. Patient had an intravenous access secured and resuscitation commenced. She was placed on intravenous antibiotics (Intravenous Ciprofloxacin and Metronidazole), a nasogastric tube size $18 \mathrm{~F}$ was passed for feeding (Figure 1) and daily wound dressing was commenced. On admission, the patient weighs $38 \mathrm{~kg}$. She was weighed daily afterwards till discharge.

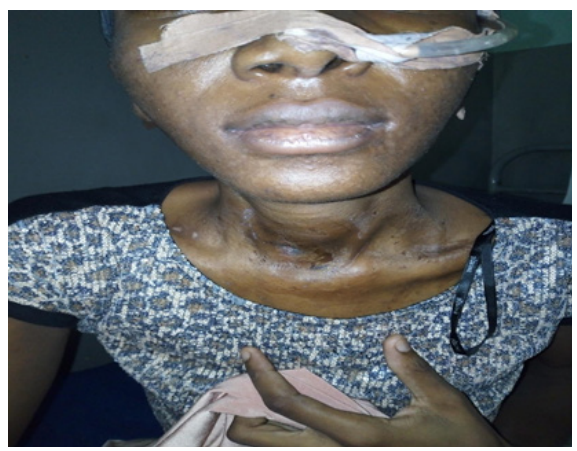

Figure 1: Patient with nasogastric tube in situ as part of conservative management.

The full blood count revealed packed cell volume of $32.1 \%$, white blood cell count of $11.9 \times 109 / \mathrm{L}$, neutrophilia of $81.6 \%$, platelets count of $141 \times 109 / \mathrm{L}$. Electrolytes, urea and creatinine revealed hyponatremia of $133 \mathrm{mmol} / \mathrm{L}$, hypokalaemia of $2.8 \mathrm{mmol} / \mathrm{L}$, urea of $4.8 \mathrm{mmol} / \mathrm{L}$, creatinine of $106 \mu \mathrm{mol} / \mathrm{L}$, chloride of $92 \mathrm{mmol} / \mathrm{L}$ and bicarbonate of $25 \mathrm{mmol} / \mathrm{L}$. Retroviral screening was negative. Barium fistulography was done after adequately counseling the patient about the procedure and consent obtained. The neck was carefully cleaned with a diluted antiseptic solution. Using a small size nasogastric tube (size 6), the cutaneous opening was canulated and about $50 \mathrm{mls}$ of barium paste was introduced, and almost $50 \mathrm{mls}$ of barium paste was ingested by the patient. Multiple lateral, oblique and antero-posterior views were taken. Antero-posterior and lateral views of barium fistulography revealed numerous fistulous tracts from the oropharynx, the largest being $13 \times 1.5 \mathrm{~cm}$ in length and width respectively projected on the anterior chest wall. The larynx and remaining parts of the airway appear intact (Figures 1, 2a, 2b).

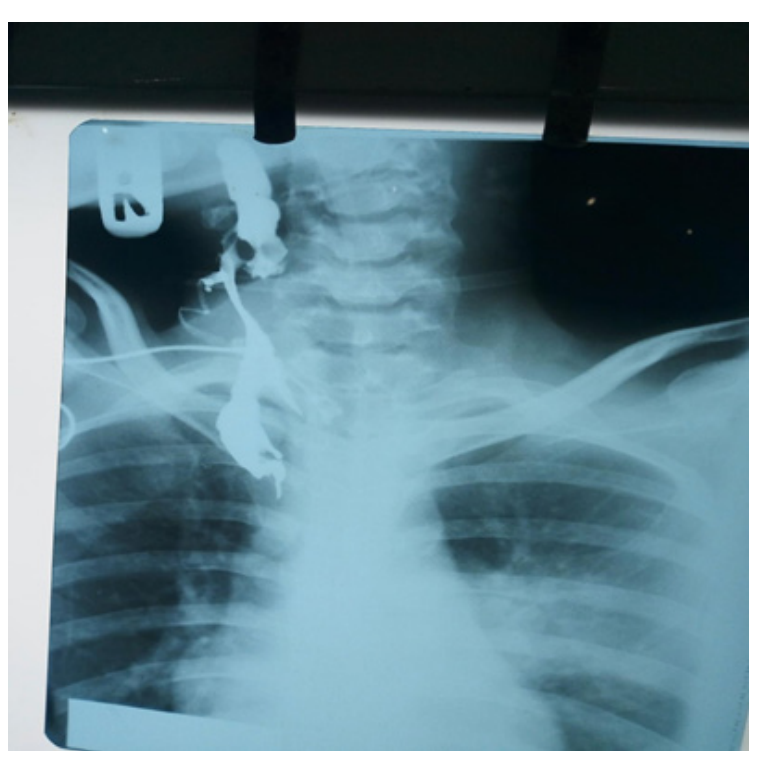

Figure 2a: Antero-posterior view of barium fistulogram of the patient showing the fistulous tract Thick Arrow showed the point of diversion of the contrast medium to the fistulous tract. Black Arrow shows the scalp vein tube used for canulating the skin opening of the fistula. 


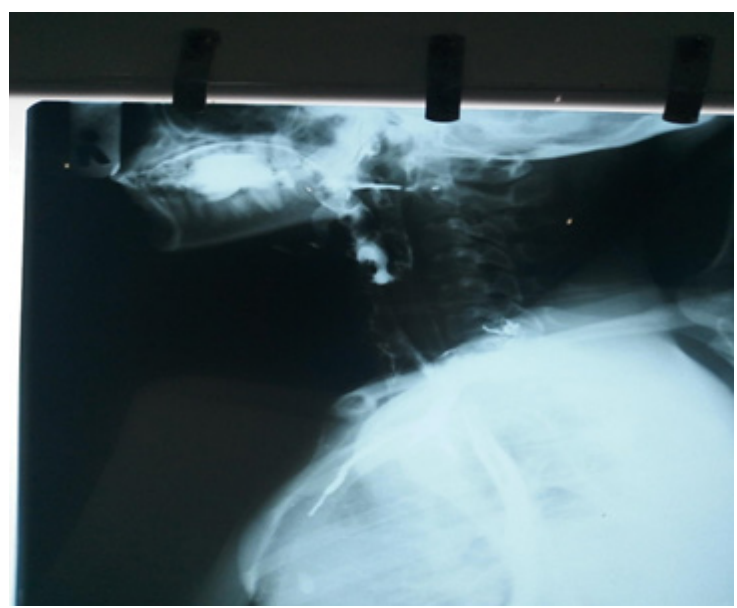

Figure 2b: Lateral view of barium fistulogram of the patient showing anterior mediastinal extension of the opacified fistulous tract. Dark Arrow shows the extension of the barium to the anterior mediastinum.

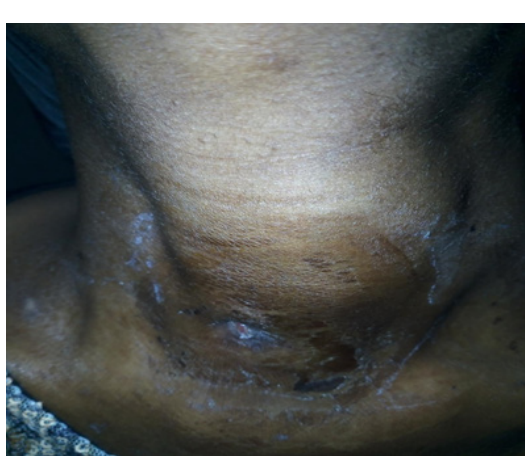

Figure 3: Healed anterior neck fistula on the day of discharge

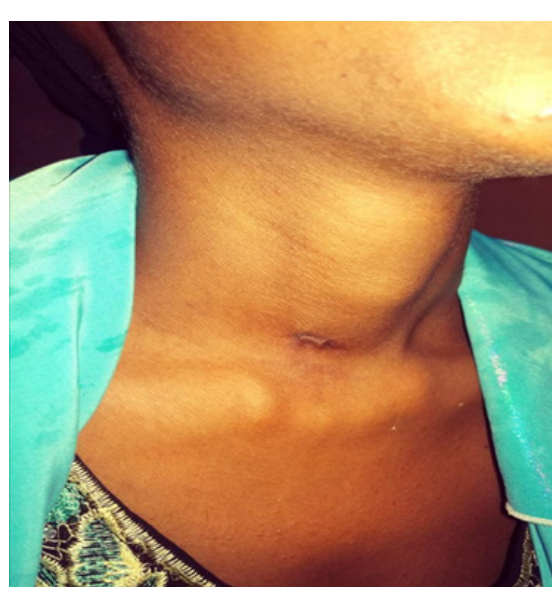

Figure 4: Healed fistulous wound six weeks after discharge.

Patient was fed via the nasogastric tube with fortified pap prepared from cereals, soyabeans, groundnuts and milk. Patient's weight improved remarkably, however the output of the fistula did not reduce remarkably, warranting a change in the antibiotic to a third-generation cephalosporin. Full strength Darrow's solution was introduced to correct the hypokalaemia. Repeat serum electrolytes analysis revealed correction of the hypokalaemia to $3.5 \mathrm{mmol} / \mathrm{L}$. On the 17th day post admission, oropharyngeal examination revealed closure of the cavity with no leakage of food contents (Figure 3) when oral feeding was attempted. Patient was commenced on peritubal feeding and monitored for the next 72 hours, antibiotics changed to oral to be served through the nasogastric tube. Patient was then discharged on 25th day post admission and counseled for interval tonsillectomy after eight weeks (Figure 3, 4). 


\section{Discussion}

The management of pharyngocutaneous fistula resulting from traditional tonsillotomy is quite challenging especially in our setting. The initial predisposing factor warranting the patient to patronize the traditional barbers usually revolves around poverty and in some cases, lack of awareness. When the index patient presented, she was malnourished, cachectic, dehydrated and weak. Upon initial assessment and institution of resuscitative measures, correction of fluid and electrolyte imbalance, treatment of infection and nutritional rehabilitation, the patient improved remarkably.

Seneviratne and Wickramsinghe [5] in Srilanka conservatively managed a fifty year old man with pharyngocutaneous fistula following peritonsillar abscess with nasogastric intubation, perenteral antibiotics and perenteral nutrition to achieve fistula closure, however on the premise of fear of complications associated with nasogastric intubation, the authors considered feeding gastrostomy as a means of trophic feeding. Our patient did very well with nasogastric intubation and perenteral antibiotics. Nasogastric intubation has been proven to be an invaluable procedure in the conservative management of pharyngocutaneous fistula from head and neck malignancies [6], as well as suppurative infections of the head and neck region $[7,8]$. We did not introduce perenteral nutrition in the conservative management despite being aware of its numerous advantages in ensuring rapid healing of fistula; this was due to financial constraints on the side of our patient. Our patient did very well with nutritional rehabilitation in form of high protein pap (pap prepared from cereal, ground nuts and soya beans) which is cheap, readily available and easy to prepare. In the index case, fistula closure was achieved within three weeks of treatment. Documented methods of managing pharyngocutaneous fistula include surgical excision of the fistulous tract after taking care of the predisposing factors such as malnutrition [9]. Pharyngocutaneous fistula arising from surgical treatment of head and neck malignancies can be prevented by meticulous surgery, adoption of negative-pressure wound closure by use of suction drainage as well as a continuous suction device [10]. Special attention should be paid to the nutritional state of patients with pharyngocutaneous fistula arising from an infective as well as neoplastic aetiologies.

It is pertinent to note that patient education goes a long way in making them present to the hospital early when sick thereby avoiding late presentation, and most often with complications. In the index case, the patient went to traditional barber for care at the first instance, there after presented to a peripheral hospital when the traditional intervention became complicated, and later on, presented to our facility. Had the patient presented to our facility earlier, time and resources wouldn't have been wasted before attaining good health. Furthermore, the complication of having a deep neck space abscess and a pharyngocutaneous fistula.

\section{Conclusion}

Pharyngocutaneous fistula from traditional tonsillotomy can be managed conservatively with nasogastric intubation, intravenous antibiotics, wound care, fluid and electrolyte replacement as well as nutritional rehabilitation. The management is multidisciplinary involving the Head and Neck Surgeon, the Radiologist, the Nutritionist and most importantly, the patient and his/her family. Patient education on early presentation to hospital is very vital in ensuring a better outcome.

\section{Acknowledgement}

None.

\section{Conflict of Interest}

No conflicts of interest.

\section{References}

1. Samdi MT, Emmanuel M, Kirfi AM (2017) Adenotonsillar surgeries in Kaduna, Nigeria. Ann Indian Acad Otorhinolaryngol Head Neck Surg 1: 6-8

2. Tshifularo M, Joseph CA, Ogunbanjo GA (2005) Post-tonsillectomy haemorrhage following traditional uvulectomy in an adult patient. S A Fam Med Pract 47(1): 46.

3. Kirfi AM, Fufore MB, Grema US, Adoga AA (2019) Management of deep neck space infections in a Nigerian Referral Center. BioClin International Research Journal 1(1): 4-9.

4. Kirfi AM, Mohammed GM, Abubakar TS, Labaran AS, Samdi MT, et al. (2014) Clinical profile and management of aerodigestive foreign bodies in North-western Nigeria. Sudan Med Monit 9: 39-43.

5. Seneviratne RW, Wickramsinghe A (2014) Pharyngo-Cutaneous fistula following drainage of a parapharyngeal abscess; a rare and challenging complication of tonsillitis. Galle Medical Journal 19 (2): 28-29.

6. Chang HP, Hong JW, Lee WJ, Kim YS, Koh YW, et al. (2018) Incorporating a Continuous Suction System as a Preventive Measure against Fistularelated Complications in Head and Neck Reconstructive Surgery. Arch Plast Surg 45: 449-457.

7. Ahmed A, Hesham S, Ahmed ME (2020) A rare case of pharyngocutaneous fistula after peritonsillar abscess. J Am Sci 16(2): 71-73.

8. Meher R, Singhal D, Girhotra M, Singh I (2002) Palatal Perforation after Tonsillectomy. The Internet Journal of Otorhinolaryngology 2(2): 1-4.

9. Kim PD (2020) Pharyngocutaneous Fistula. In: Kountakis SE (Ed.) Encyclopedia of Otolaryngology, Head and Neck Surgery. Springer, Berlin, Heldelberg, Germany.

10. Loaec E, Vaillant PY, Bonne L, Marianowski R (2014) Negative-pressure wound therapy for the treatment of pharyngocutaneous fistula. Eur Ann Otorhinolaryngol Head Neck Dis 131: 351-355. 